\title{
Persönlichkeitsverletzung durch eine Medienkampagne
}

Kommentar zu BGE 143 III 297 (Hirschmann II)

Bettina Bacher *

In einem neuen Grundsatzurteil hat das Bundesgericht erstmals eine Persönlichkeitsverletzung durch eine Medienkampagne festgestellt. Der vorliegende Aufsatz stellt Tatbestandsmerkmale, Rechtfertigungsgründe und Rechtsfolgen einer solchen Persönlichkeitsverletzung dar und ordnet den Entscheid in einen grösseren Kontext ein.

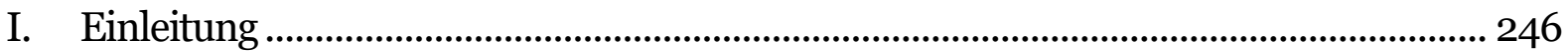

II. Sachverhalt und Zusammenfassung der Erwägungen................................................... 246

1. Sachverhalt und Prozessgeschichte ......................................................246

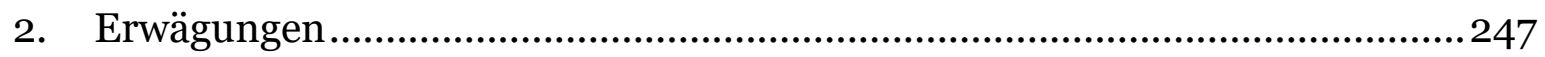

III. Medienkampagne als Persönlichkeitsverletzung - Versuch der Verallgemeinerung 250

1. Tatbestandsmerkmale .............................................................................250

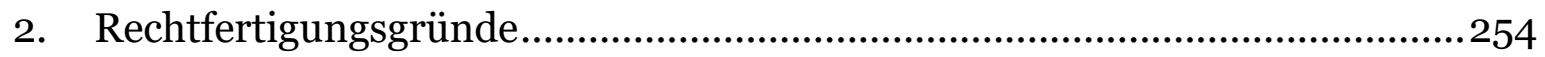

3. Negatorische Klagen..................................................................................256

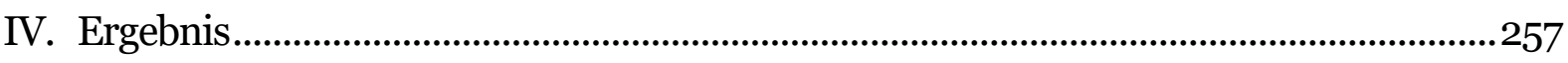

V. Schlusswort .................................................................................................................259

Zitiervorschlag: Bettina Bacher, Persönlichkeitsverletzung durch eine

Medienkampagne, in: sui-generis 2017, S. 245

URL: $\quad$ sui-generis.ch/47

DOI: $\quad$ https://doi.org/10.21257/sg.47

* Dr. iur. Bettina Bacher, Rechtsanwältin, Lehrbeauftragte und Lektorin an der Universität Fribourg, Wissenschaftliche Mitarbeiterin beim Bundesamt für Justiz. 


\section{Einleitung}

1 Die Rechtsstreitigkeiten rund um Carl Hirschmann beschäftigen die Gerichte schon seit Langem. Das Verfahren wurde deswegen auch als «Monsterprozess» bezeichnet. ${ }^{1}$ Anfang Juni 2017 hat das Bundesgericht nun ein weiteres Urteil gefällt. ${ }^{2}$ Es äusserte sich darin ein zweites Mal zu persönlichkeitsverletzenden Medienberichten, die insbesondere im $\mathrm{Zu}-$ sammenhang mit der Verhaftung von Carl Hirschmann im November 2009 veröffentlicht wurden (vgl. zum Sachverhalt unten II.). Das Urteil führte in ersten Einschätzungen bereits zu kontroversen Diskussionen. Schwaibold, seinerseits häufiger Vertreter der Medienseite (allerdings nicht in diesem Verfahren), bezeichnete es als «Katastrophe». 3 Anderer Ansicht war Glasl, Vertreter der Klägerseite im vorliegenden Verfahren, in seiner Replik auf diese Urteilsbesprechung. 4

Canonica Simon, 25 Jahre medienrecht: Konstanten und Variablen, Medialex 2016 S. 3 ff., S. 5.

Urteil des Bundesgerichts 5A_256/2016 vom 9. Juni 2017, inzwischen auszugsweise publiziert als BGE 143 III 297 (nachfolgend: Hirschmann II). Der erste Entscheid ist das Urteil des Bundesgerichts 5A_658/2014 vom 6. Mai 2015 (nachfolgend: Hirschmann I). Dagegen wurde erfolglos ein Revisionsgesuch gestellt (Urteil des Bundesgerichts 5F_8/2015 vom 31. August 2015). Carl Hirschmann seinerseits wurde ebenfalls wegen Verletzung der Persönlichkeit verurteilt (Urteil des Bundesgerichts 5A_309/2013 vom 4. November 2013). Auch das Strafverfahren gegen Carl Hirschmann ging bis vor Bundesgericht (Urteil des Bundesgerichts 6B_215/2013 vom 27. Januar 2014 sowie die Revision in Urteil des Bundesgerichts 6B_1222/2014 vom 20. Januar 2015); er wurde wegen sexueller Nötigung, mehrfacher sexueller Handlungen mit einem Kind, mehrfacher versuchter Nötigung sowie Tätlichkeiten zu einer Freiheitsstrafe von 32 Monaten, wovon 20 Monate bedingt, und einer Busse von Fr. 4000.- verurteilt.

3 Schwaibold Matthias, Ein Schrecken ohne Ende, Medialex 6/2017, N 3.

4 Glasl Daniel, Medienkampagne und Gewinnabschöpfung - kein Schrecken ohne Ende, Medialex 7/8/2017.
2 In Hirschmann I setzte sich das Bundesgericht zu einem grossen Teil mit verfahrensrechtlichen Fragestellungen auseinander. In Bezug auf den zivilrechtlichen Persönlichkeitsschutz bot der Entscheid nicht viel Neues. In Hirschmann II erfolgten hingegen einige interessante Weiterentwicklungen im Bereich des zivilrechtlichen Persönlichkeitsschutzes. Insbesondere hielt das Bundesgericht fest, die Beklagten hätten durch eine Medienkampagne die Persönlichkeit des Klägers verletzt. Die vorliegende Untersuchung beschäftigt sich mit den Tatbestandsmerkmalen, der Rechtfertigung und den Rechtsfolgen der «Persönlichkeitsverletzung durch eine Medienkampagne» und unternimmt den Versuch, diese Punkte $\mathrm{zu}$ verallgemeinern. Nicht behandelt werden hingegen die Erwägungen zur Gewinnherausgabe. Sie verdienten eine eigene Untersuchung, zumal aus verfahrensrechtlicher Sicht.

\section{Sachverhalt und Zusammen- fassung der Erwägungen}

\section{Sachverhalt und Prozessgeschichte}

3 Carl Hirschmann (nachfolgend: der Kläger) ist Unternehmer und leitete bis Ende 2010 einen Club in Zürich. Er verklagte am 24. Februar 2011 die Unternehmen Tamedia AG, 20 Minuten AG, 20 Minutes Romandie SA sowie Espace Media AG vor dem Handelsgericht des Kantons Zürich und machte eine Verletzung der Persönlichkeit durch die Berichterstattung der Beklagten geltend. Im Vordergrund stand die Berichterstattung über die Verhaftung des Klägers am 3. November 2009 (er wurde am 6. November 2009 wieder entlassen). Hauptsächlich ging es um Zeitungsberichte, daneben auch um 
Beiträge des Fernsehsenders TeleZüri und des Radiosenders Radio 24 sowie Veröffentlichungen auf den OnlineNewsportalen der Beklagten. Die Berichte betrafen angebliche Sexualdelikte des Klägers, angebliche Erpressungen, Nötigungen, Drohungen, Freiheitsberaubungen, angebliche physische Gewalt insbesondere gegenüber Frauen sowie angebliche Charakterschwächen, sittenwidriges Verhalten oder psychische Krankheit.5 Dabei sei der Kläger mit vollem Namen genannt worden. Darüber hinaus rügte der Kläger, durch die Verlinkung der zahlreichen Berichte, Videos und Radiobeiträge hätten die Beklagten eine Medienkampagne gegen ihn geführt.

4 Der Kläger verlangte einerseits die Feststellung einer widerrechtlichen Persönlichkeitsverletzung und das Verbot einer Weiterverbreitung der beanstandeten persönlichkeitsverletzenden Inhalte. Andererseits beantragte er die zweimalige Veröffentlichung des Urteilsdispositivs, die Löschung sämtlicher persönlichkeitsverletzender Inhalte auch aus sämtlichen Archiven und Internetsuchmaschinen (Google, inkl. Google-Index und GoogleCache). Darüber hinaus forderte er Schadenersatz, Genugtuung und Herausgabe des Gewinns, den die Beklagten mit den Berichten bzw. der Medienkampagne gemacht hätten.

5 Das Handelsgericht Zürich hiess die Klage am 26. Juni 2014 teilweise gut, verneinte aber die eingeklagten Persönlichkeitsverletzungen mehrheitlich und wies die Begehren um Schadenersatz, Genugtuung und Gewinnherausgabe ab. Der

5 Vgl. die nach Themenbereichen geordneten Rechtsbegehren im Sachverhalt in Hirschmann I (Fn. 2).
Kläger wandte sich daraufhin an das Bundesgericht, das die Beschwerde am 6. Mai 2015 teilweise guthiess und die Sache zur Neubeurteilung an das Handelsgericht zurückwies (Hirschmann I). Das Handelsgericht fällte am 8. Februar 2016 ein neues Urteil, das der Kläger wiederum vor Bundesgericht anfocht. Dieses hiess die Beschwerde des Klägers am 6. Juni 2017 teilweise gut und wies die Sache erneut an das Handelsgericht Zürich zurück (Hirschmann II).

\section{Erwägungen}

6 Der Kläger verlangte die Feststellung, dass die Beklagten «durch ihre Berichte (Artikel, Bilder, Videos, Radiosendungen; jeweils unter voller Namensnennung) und deren permanente Verlinkung» in 9 Medien (print und online) eine «eigentliche Medienkampagne» gegen ihn geführt haben und ihn damit widerrechtlich in seiner Persönlichkeit verletzt haben. Das Bundesgericht geht vom Zeitraum vom 4. November 2009 bis zum 2. Oktober 2010 aus. ${ }^{6}$ Die genaue Anzahl Berichte, um die es dabei geht, bleibt jedoch unklar; die Rede ist von 120 bis 140 Berichten. Jedenfalls handelt es sich um eine Vielzahl von Berichten, wobei teilweise täglich mehrere Berichte über den Kläger veröffentlicht worden seien. Dies erfolgte über verschiedene Medien, d.h. über Zeitungen, Online-Medien, Newsportale, Radio und Fernsehsender, wobei die Berichte teilweise miteinander verlinkt wurden. Insgesamt könne der «Medienhype» als präzedenzlos erachtet werden, der Fall sei geradezu medial ausgeschlachtet worden.7

\footnotetext{
Vgl. Hirschmann II, E. 6.5.

HGer ZH HG150112-O vom 8. Februar 2016 E. 5.6.3.
} 
7 Das Bundesgericht beurteilte diese Vorgänge gesamthaft als Persönlichkeitsverletzung durch eine Medienkampagne. Es beginnt in E. 6.4.1 mit grundlegenden Ausführungen zur Persönlichkeit als «Gesamtheit des Individuellen». Die persönlichen Verhältnisse erfassten nicht nur die Person selbst, sondern auch Tatsachen der körperlichen und gesellschaftlichen Aussenwelt, die Persönlichkeit sei also ein gesellschaftlicher Beziehungsbegriff.

Weiter hält das Gericht in E. 6.4.2 fest, das Persönlichkeitsrecht verschaffe seinem Träger die Befugnis, über die persönlichen Güter grundsätzlich frei von fremder Einwirkung, wie z.B. Presseäusserungen, zu herrschen. Vorliegend seien die Persönlichkeitsgüter der Privatsphäre und der Ehre «berührt», wodurch eine Persönlichkeitsverletzung gegeben sein könne, selbst wenn die Berichterstattung die Wahrheit wiedergebe. Ausschlaggebend sei nicht der Wahrheitsgehalt oder die Objektivität medialer Äusserungen, sondern ob die Berichte in die Geheim- oder Privatsphäre eingreifen oder die betroffene Person auf unzulässige Weise in ihrem Ansehen herabsetzen. Das gelte auch in Bezug auf die Unschuldsvermutung. Jede allein von ihrem Gegenstand her erlaubte Presseäusserung finde ihre Grenze im Recht des Einzelnen auf Achtung der Privatsphäre, denn dieser brauche sich eine dauernde Beobachtung nicht gefallen zu lassen. Jeder solle innerhalb gewisser Grenzen selbst bestimmen dürfen, wer was über ihn wisse und was über ihn im Verborgenen bleiben solle. Von diesem legitimen Diskretionsbedürfnis seien Publikationen beliebiger Art erfasst, die «einen im Einzelfall zu konkretisierenden schutzwür- digen Bereich des Privaten nicht respektieren», nicht nur solche, die das Ansehen einer Person in der Öffentlichkeit beeinträchtigten. So schütze die informationelle Privatheit in einem weiten Sinne überall dort, wo der Einzelne durch eine Wiedergabe von Informationen in seiner Persönlichkeit tatsächlich und spürbar beeinträchtigt werde.

9 In E. 6.4.3 beschäftigt sich das Gericht mit dem persönlichkeitsverletzenden Verhalten. Die Verletzung könne in einem Tun, Dulden oder Unterlassen bestehen, in der Form eines einmaligen Aktes, von Wiederholungshandlungen oder eines Zustandes erfolgen und das Medium sei unerheblich. Erforderlich sei eine gewisse Intensität. $\mathrm{Zu}$ beachten sei bezüglich Presseäusserungen, dass diese einen ungleich grösseren Personenkreis erreichten als private Äusserungen und auch später erneut zur Kenntnis genommen werden könnten. So verschiebe sich die Grenze zwischen Gemein- und Privatbereich zugunsten des von einer Presseäusserung Betroffenen. Gerade heutzutage liessen sich selbst harmlose Informationen zu einem schützenswerten Persönlichkeitsprofil verdichten.

10 In E. 6.5 kommt das Bundesgericht zu seinen Schlussfolgerungen in casu. Entgegen der Ansicht der Vorinstanz sei das Vorliegen einer persönlichkeitsverletzenden Berichterstattung nicht alleine daran zu messen, welchen Anschein diese beim Durchschnittsleser unter dem Blickwinkel des Wahrheitsgehalts oder der Unschuldsvermutung erweckten. "Losgelöst von diesen Prüfungskriterien» rühre das, was vorgefallen sei (d.h. der präzedenzlose Medienhype) an das Recht des Beschwerdeführers auf Achtung seiner in- 
formationellen Privatsphäre. Trotz der Stellung des Klägers als BoulevardProminenter sprenge die Medienkampagne den Rahmen dessen, was dieser sich gefallen lassen müsse. Die vorinstanzliche Feststellung, dass die Medien in überdurchschnittlicher Intensität berichtet und sich regelrecht auf das Ereignis gestürzt hätten, lasse keinen anderen Schluss zu, als dass die Beklagten den Beschwerdeführer in seiner Persönlichkeit verletzt hätten, indem sie ihn seines privaten Herrschaftsrechts beraubten, selbst darüber zu bestimmen, welche Informationen die Öffentlichkeit über sein Leben erfahren soll. Selbst wenn sie in Bezug auf das laufende Verfahren objektiv keinen falschen Eindruck erweckt haben sollten, hätten die Berichte Begebenheiten und Ereignisse aus dem Leben des Klägers preisgegeben in einer Weise, die einer «übermässigen Einmischung in die Individualität» gleichkomme und den Kläger in den Augen des Durchschnittskonsumenten blossstelle.

11 Das Bundesgericht prüft die Rechtfertigung in E. 6.7. Das Gericht hält in E. 6.7.2 fest, dass ein Interesse einer weiteren Öffentlichkeit am Milieu, in dem der Kläger in Erscheinung getreten sei, unbestritten vorliege. Zwar können nach $\mathrm{E}$. 6.7.3 auch Sensationsberichte, mit denen ein Boulevardblatt oder ein Lokalfernsehen seinem Publikum die Zeit vertreibe, im «öffentlichen (Unterhaltungs-) Interesse» liegen. Falls sich eine skandalisierende Berichterstattung aber als persönlichkeitsverletzend erweise, stelle sich die Frage, ob «ein reines Unterhaltungsbedürfnis im Streit unter Privaten, das heisst nach privatrechtlichen Massstäben» das Interesse des Verletzten auf Achtung seiner Privatsphäre mindestens aufwiege. Bei dieser Abwägung komme der reinen Unterhaltung als öffentlichem Interesse nicht das Gewicht des Informierens zu, «verstanden als aufklärendes Vermitteln von Neuigkeiten aus verschiedensten Bereichen des Allgemeininteresses». «Je weiter die reine Unterhaltung als von den Medien bedientes Bedürfnis in den Vordergrund rückt, desto schwerer fällt es, ein (überwiegendes) öffentliches Interesse anzunehmen.»

12 Im konkreten Fall erblickt das Bundesgericht kein nennenswertes Informationsbedürfnis, da es bei der Medienkampagne nicht darum gegangen sei, dem Publikum Klarheit über den Fortgang des Strafverfahrens zu verschaffen, sondern darum sich am Medienrummel zu beteiligen, indem eine Vielzahl von Berichten zum immer gleichen Thema publiziert wurde. Diese Art der Berichterstattung vermöge die Persönlichkeitsverletzung des Klägers nicht aufzuwiegen. Ein wirtschaftliches (privates) Interesse der Beklagten ist nach Ansicht des Bundesgerichts ebenfalls erstellt; ob dieses als Rechtfertigungsgrund dienen kann, bleibt in E. 6.7.4 aber offen. Im Ergebnis stellt das Bundesgericht die Persönlichkeitsverletzung fest.

13 Der Kläger erhebt weiter eine Beseitigungsklage. Die Artikel, die Bestandteil der Medienkampagne gewesen seien, seien weiterhin im Internet verfügbar, wodurch der Störungszustand fortbestehe. Das Bundesgericht führt in E. 7.4.2 aus, der Kläger schiesse am Ziel vorbei, wenn er mit seinem Beseitigungsanspruch eine Vielzahl unspezifizierter Medienberichte ins Visier nehme, denn «die Persönlichkeitsverletzung, gegen die sich sein allfälliger Beseitigungsanspruch 
richten würde, ist die Medienkampagne selbst». Dass sich die damaligen Medienberichte heute noch abrufen lassen, bedeute nicht, dass auch der damals entbrannte Medienhype nach wie vor andauere und damit eine bestehende Persönlichkeitsverletzung im Sinne von Art. 28a Abs. 1 Ziff. 2 ZGB vorliege. Der Kläger könne nicht «auf einen Streich und ohne weitere Begründung» sämtliche Medienberichte aus der Welt schaffen, die in seinen Augen sein Recht auf Privatleben beeinträchtigten. Das Gericht weist deswegen die Beseitigungsklage ab.

\section{Medienkampagne als Persönlich- keitsverletzung - Versuch der Verallgemeinerung}

14 Soweit ersichtlich ist die Persönlichkeitsverletzung durch eine Medienkampagne eine Premiere in der bundesgerichtlichen Rechtsprechung. Mit Blick auf den konkreten Fall mag das Urteil grundsätzlich stimmig erscheinen. Seine Bedeutung und Einordnung hängen indes davon ab, ob eine tragfähige Verallgemeinerung gelingt. Dies wird nachfolgend in Bezug auf die drei zentralen Aspekte versucht: die Tatbestandsmerkmale für das Vorliegen einer Persönlichkeitsverletzung durch eine Medienkampagne, allfällige Rechtfertigungsgründe und schliesslich mögliche Rechtsfolgen, wobei ich mich auf die negatorischen Klagen beschränke.

\section{Tatbestandsmerkmale}

15 Das Bundesgericht hält die Tatbestandsmerkmale einer Persönlichkeitsverletzung durch eine Medienkampagne abstrakt nicht ausdrücklich fest; vielmehr müssen sie aus den verschiedenen Erwägungen herausgefiltert werden. So dürfte eine Persönlichkeitsverletzung durch eine Medienkampagne vorliegen, wenn (1) über einen längeren Zeitraum (2) mit relativ hoher Intensität (3) zu einer bestimmten Person Medienberichte mit einem engen thematischen Fokus publiziert werden und (4) diese dadurch tatsächlich und spürbar in ihrer Persönlichkeit beeinträchtigt wird. Zentral ist, bei der Formulierung der Tatbestandsmerkmale nicht bereits eine wertende Abwägung vorzunehmen. Denn diese gehört zur Rechtfertigung einer Persönlichkeitsverletzung.

\section{a) Berichterstattung über einen längeren Zeitraum}

16 Festzustehen scheint zunächst, dass eine Medienkampagne nur vorliegen kann, wenn über einen längeren Zeitraum berichtet wird. Vorliegend geht das Bundesgericht davon aus, dass der Medienhype knapp 11 Monate angedauert hat. ${ }^{8}$ Ganz allgemein dürfte von einem längeren Zeitraum - und damit von einer Medienkampagne - erst die Rede sein, wenn sich die fragliche Berichterstattung zumindest über mehrere Wochen, eher aber Monate hinzieht. Daraus ergibt sich umgekehrt, dass eine kurzzeitige intensive Berichterstattung, wie sie in den Medien häufig stattfinden dürfte, noch keine Medienkampagne darstellt.

\section{b) Hohe Intensität der Berichterstattung}

17 Weiter ist die hohe Intensität der Berichterstattung ein zentrales Element für das Vorliegen einer Medienkampagne. Im zu beurteilenden Sachverhalt wurden teilweise mehrmals täglich weitere Be-

$8 \longdiv { \text { Mangels verbindlicher Sachverhaltsermittlungen } }$ der Vorinstanz bleibt offen, ob die Kampagne weitergeführt wurde; vgl. E. 6.6. 
richte veröffentlicht. Die konkrete Anzahl der Berichte, die Teil der Kampagne sein sollen, bleibt aber offen, obwohl dies für eine Einschätzung der Intensität über die Zeitdauer durchaus interessant wäre. Es ist aber davon auszugehen, dass diese Berichte zeitweilig zu einer prägenden Medienpräsenz des Klägers führten, indem täglich von ihm die Rede war.

Unerheblich scheint für das Bundesgericht, wo die Berichte platziert waren (Frontseite etc.). Ebenfalls unklar ist, ob die Berichterstattung während des gesamten Zeitraums von gleichbleibender Intensität war. Sollte die Zahl von 140 Berichten zutreffen, liesse dies darauf schliessen, dass während der 11 Monate nicht täglich mehrere neue Berichte publiziert wurden. Das Urteil bleibt in diesem Punkt relativ vage.

So bleibt es schwierig zu definieren, wann eine Berichterstattung die Schwelle der hohen Intensität überschreitet und zur Medienkampagne wird. Die hohe Intensität ist ein rein quantitatives Kriterium. Wendet man es völlig losgelöst vom Inhalt der Berichterstattung an, wird nicht genügend berücksichtigt, dass Medien oftmals über einen längeren Zeitraum einen bestimmten Sachverhalt und dessen Entwicklung verfolgen. So wäre es seltsam, wenn beispielsweise die Berichterstattung über ein Unglück, die komplizierte Suche nach dessen Opfern und deren weiteres Schicksal, die sich über längere Zeit hinzieht, als Kampagne betrachtet würde. Deswegen reicht dieses Kriterium alleine noch nicht aus, um eine Kampagne festzustellen.

Das Bundesgericht geht nicht darauf ein, ob die Kampagne, wie der Kläger geltend gemacht hatte, durch die Verlinkung der verschiedenen Berichte ausgelöst oder zumindest mitausgelöst wurde. Die Verlinkung ist damit kein entscheidendes Kriterium für das Vorliegen einer Kampagne. Das erscheint zutreffend. Andernfalls würde nämlich bald jede OnlineBerichterstattung zur Kampagne, weil es heutzutage zum Standard gehört, Berichte zu einem Thema miteinander zu verlinken oder ein Online-Dossier anzulegen. Massgebend muss daher die regelmässige erneute Publikation von Berichten sein. Darunter kann aber auch das Aktualisieren oder Wiederverwerten bestehender Artikel fallen, z.B. indem diese erneut auf der Frontseite oder am Anfang einer Website platziert werden.

\section{c) Personenberichterstattung mit engem thematischem Fokus}

21

Damit eine Medienkampagne vorliegt, muss die fragliche Berichterstattung über eine Person inhaltlich einen relativ engen Fokus aufweisen. Im vorliegenden Sachverhalt konzentrierte sich die Berichterstattung auf den Kläger und seine Verhaftung, d.h. ein paar wenige, eng zusammenhängende Themenkreise. Beide Aspekte dürften gleichermassen relevant sein. Allein weil regelmässig über eine bestimmte Person berichtet wird, kann noch keine Kampagne vorliegen; man denke nur an Bundesräte oder Sportler. Vielmehr muss sich die Berichterstattung zu einer Person wiederholt auf einen bestimmten Sachverhalt oder eine eng umrissene Thematik beziehen. Allerdings zeigen sich rasch Abgrenzungsschwierigkeiten, beispielsweise wenn regelmässig über einen bestimmten Aspekt einer Person, wie ihre Frisur, berichtet wird. So wäre es fragwürdig, wenn regelmässige Berichte über die neueste Frisur einer 
Schauspielerin als Kampagne betrachtet würden.

In Bezug auf dieses Kriterium muss hingegen irrelevant bleiben, ob an den Inhalten der Kampagne bzw. an einer fraglichen Information überhaupt ein Interesse besteht oder ob sie z.B. lediglich der Unterhaltung dienen. Diese Frage ist erst bei der Interessenabwägung zur Rechtfertigung einer Persönlichkeitsverletzung zu prüfen. Sie kann daher nicht zugleich als Begründung für das Bestehen einer Persönlichkeitsverletzung herangezogen werden. Es geht mithin nicht darum, das Informationsinteresse $\mathrm{zu}$ prüfen, sondern festzustellen, ob zwischen den fraglichen Beiträgen ein genügend enger inhaltlicher Zusammenhang besteht, um von einer Kampagne zu sprechen.

\section{d) Tatsächliche und spürbare Beeinträchtigung der Persönlichkeit}

23 Die persönlichkeitsverletzende Qualität, welche das Bundesgericht in der Medienkampagne erblickt, macht es an einer Verletzung der Befugnis fest, über die persönlichen Güter grundsätzlich frei von fremder Einwirkung zu herrschen. 9 Dabei spricht das Bundesgericht zwar vom Recht auf Achtung der Privatsphäre. Aber es versteht diese nicht im Sinne der Sphärentheorie, wonach Informationen abhängig von ihrer Nähe zu einer Person verschiedenen Sphären zugeordnet werden. ${ }^{10}$ Demnach ist der Schutz umso um-

\footnotetext{
Vgl. zum Ganzen E. 6.4.2.

10 Meili Andreas, Kommentar zu Art. 28 ZGB, in: Honsell Heinrich/Vogt Nedim Peter/Geiser Thomas (Hrsg.), Basler Kommentar, Zivilgesetzbuch I, Art. 1-456 ZGB, 5. Aufl., Basel 2014, Art. 28 ZGB N 23; Bacher Bettina, Medienfreiheit und Persönlichkeitsschutz, Zivilrechtliche Auswirkungen der Lösung eines Grundrechtskon-
}

fassender, je persönlichkeitsnäher eine Information ist: die Gemeinsphäre ist kaum geschützt, die Privat- und v.a. die Geheimsphäre hingegen intensiv. Sowohl der Schutz als auch der Eingriff richten sich nach relativen Kriterien.

24 Vorliegend versteht das Bundesgericht die Achtung der Privatsphäre hingegen im Sinne der informationellen Selbstbestimmung. Sie gewährt grundsätzlich ein individuelles Selbstbestimmungs- und Herrschaftsrecht über persönliche Informationen. Dieses gilt unabhängig vom Inhalt und damit auch von der Persönlichkeitsnähe der fraglichen Informationen. Der Schutz ist mithin nicht relativ, so dass sich auch der Eingriff nicht nach relativen und damit inhaltsbezogenen Kriterien bestimmt. Vielmehr liegt in jeder fremden Einwirkung, d.h. in jeder Beeinträchtigung des persönlichen Herrschaftsrechts, zugleich ein Eingriff. Diese Konstruktion liegt dem Datenschutzgesetz zugrunde. ${ }^{11}$ Jede Bearbeitung stellt aus dieser Perspektive grundsätzlich ein Eingriff dar, der eines Rechtfertigungsgrundes bedarf.

25 Allerdings ist das Konzept der informationellen Selbstbestimmung umstritten. Im Bereich des Verfassungsrechts legen die Rechtsprechung und die (wohl) herrschende Lehre Art. 13 Abs. 2 BV zwar in diesem Sinne aus. ${ }^{12}$ Es gibt aber Stimmen, welche weniger weit gehen und die Norm lediglich als Missbrauchsverbot

flikts, Diss. Fribourg, Basel 2015, N 693.

11 Vgl. BGE 138 II 346, auf den das Bundesgericht in E. 6.4.2 f. verweist.

12 BGE 138 II 346 E. 8.2; BGE 136 II 508 E. 6.3.1; statt vieler Diggelmann Oliver, Kommentar zu Art. 13 BV, in: Waldmann Bernhard/Belser Eva Maria/Epiney Astrid (Hrsg.), Basler Kommentar, Bundesverfassung, Basel 2015, Art. 13 BV N 33 m.w.H. 
verstehen. ${ }^{13}$ Auch im Bereich des Datenschutzes stellt sich die Frage, ob es in Anbetracht allgegenwärtiger Datenbearbeitungen noch sinnvoll ist, jede einzelne mechanisch als Persönlichkeitsverletzung zu betrachten, die anschliessend ebenso mechanisch mit einem Rechtfertigungsgrund legitimiert wird. Es besteht die Gefahr, dass sowohl der Begriff der Verletzung als auch die Bedeutung der Rechtfertigung ausgehöhlt werden, weil sie zu alltäglichen Vorgängen werden.

Die informationelle Selbstbestimmung ist im Kontext von Art. 28 ZGB nicht völlig fremd. So gewährleistet das Recht am eigenen Bild, das auch durch Art. 28 ZGB geschützt wird, die Selbstbestimmung in Bezug auf die Veröffentlichung von Bildern. ${ }^{14}$ Diese Verfügungshoheit der betroffenen Person besteht unabhängig vom Inhalt des Bildes. ${ }^{15}$

Das vorliegende Urteil erweitert nun den Anwendungsbereich der informationellen Selbstbestimmung in Bezug auf Art. 28 ZGB. Dabei versucht das Bundesgericht, Kritik in der Lehre Rechnung zu tragen. So wird die Ansicht vertreten, die informationelle Selbstbestimmung schiesse über das Ziel des Persönlichkeitsschutzes hinaus, indem jeder noch so harmlose Eingriff einer Rechtfertigung bedürfe. ${ }^{16}$ Art. 28 ZGB setze vielmehr den Nachweis einer mehr als harmlosen

13 Belser Eva Maria/Waldmann Bernhard, Grundrechte II, Die einzelnen Grundrechte, Zürich 2012, Kap. 2 N 87; Gächter Thomas/Egli Philipp, Informationsaustausch im Umfeld der Sozialhilfe, Jusletter 6. September 2010, N 18 ff., N 24 insbes.

14 BGE 138 II 346 E. 8.2; BGE 127 III 481 E. 3b.

15 Vgl. Bacher (Fn. 10), N 530, 697 ff.

16 Hausheer Heinz/Aebi-Müller Regina, Das Personenrecht des Schweizerischen Zivilgesetzbuches, 4. Aufl., Bern 2016, N 12.124.
Beeinträchtigung voraus. Deswegen sei das Bedürfnis des Einzelnen nach informationeller Privatheit («Privatsphäre» i.S.v. Diskretion) nur soweit zu schützen, «als der Einzelne durch eine bestimmte Verhaltensweise [...] in seiner Persönlichkeit tatsächlich und spürbar beeinträchtigt» werde. ${ }^{17}$ Diese Formulierung übernimmt das Bundesgericht in E. 6.4.2.

28 Das Bundesgericht führt damit im Kontext von Art. 28 ZGB nicht einfach generell die informationelle Selbstbestimmung ein, wonach jeder Eingriff in dieselbe zugleich eine Persönlichkeitsverletzung darstellen soll. Vielmehr wird eine tatsächliche und spürbare Beeinträchtigung durch den Eingriff gefordert.

29 In der Subsumtion in E. 6.5 erblickt das Bundesgericht eine solche Beeinträchtigung darin, dass durch die intensive Berichterstattung in der fraglichen Zeit die Beklagten den Kläger seines privaten Herrschaftsrechts beraubten, selbst darüber zu bestimmen, welche Informationen über ihn öffentlich seien. In der Medienkampagne liege eine «übermässige Einmischung in die Individualität» des Klägers und dieser sei dadurch blossgestellt worden. Massgebend ist damit im Bereich von Art. 28 ZGB nach wie vor, welche konkreten Auswirkungen eine bestimmte Handlung auf die betroffene Person hat und nicht - wie im Bereich des Datenschutzes - die Handlung an sich, unabhängig von ihren Auswirkungen.

17 Hausheer/Aebi-Müller (Fn. 16), N 12.125 (Hervorhebungen im Original). 


\section{e) Und der Inhalt?}

30 Der Gegenstand der Kampagne bzw. der fraglichen Berichte scheint für das Vorliegen einer Persönlichkeitsverletzung durch eine Medienkampagne irrelevant. Die Inhalte müssen für sich alleine nicht persönlichkeitsverletzend sein, damit eine persönlichkeitsverletzende Medienkampagne vorliegt. Dies ist konsequent. Andernfalls bliebe unklar, worin der spezifische Gehalt einer persönlichkeitsverletzenden Medienkampagne liegen sollte, wenn bereits die konkreten Berichte eine Persönlichkeitsverletzung darstellten.

\section{Rechtfertigungsgründe}

31 Nachdem erstellt ist, dass eine Persönlichkeitsverletzung durch eine Medienkampagne vorliegt, ist als Nächstes zu prüfen, ob diese durch einen Rechtfertigungsgrund legitimiert ist. Es gilt damit nachzuweisen, dass ein Rechtfertigungsgrund vorliegt, um über einen längeren Zeitraum mit relativ hoher Intensität $\mathrm{zu}$ einer bestimmten Person Medienberichte mit einem engen thematischen Fokus zu publizieren und diese dadurch tatsächlich und spürbar in ihrer Persönlichkeit zu beeinträchtigen.

Grundsätzlich kommen dabei die üblichen Rechtfertigungsgründe nach Art. 28 Abs. 2 ZGB zur Anwendung: eine Einwilligung, ein überwiegendes öffentliches oder privates Interesse oder eine gesetzliche Vorschrift. Eine Gesetzesnorm zur Rechtfertigung einer Medienkampagne dürfte indes wohl kaum zu finden sein. Theoretisch denkbar ist eine Einwilligung. Im Vordergrund wird jedoch wie im vorliegenden Urteil die Interessenabwägung stehen.

\section{a) Gegenüberstellung der Interessen}

33 Wie bei jeder Abwägung sind zunächst die sich gegenüberstehenden Interessen darzulegen. Zur Rechtfertigung der Persönlichkeitsverletzung ist ein überwiegendes Interesse nötig. Dies ist der Fall, wenn die Einschränkung auf Seiten des Opfers gegenüber dem Vorteil, den einer anderen Person oder der Öffentlichkeit durch die Verletzung entsteht, als weniger bedeutsam erscheint. ${ }^{18}$

34 Auf Seiten des Klägers steht das Interesse an der Unversehrtheit seiner Persönlichkeit sowie an der Wahrung der Entscheidungsfreiheit über Informationen zu seiner Person. Da vorliegend die Persönlichkeitsverletzung auf einer Beschränkung dieser Entscheidungsfreiheit beruht, steht dieser zweitgenannte Aspekt im Vordergrund. So spricht das Bundesgericht in E. 6.7.2 vom Verlust der Privatsphäre aufgrund des Umfangs und der Intensität der Berichterstattung in ihrer Gesamtheit.

35 Auf Seiten der beklagten Medien steht ganz allgemein das Interesse an der ungehinderten Ausübung der Kommunikationsgrundrechte. Darunter fällt auch die freie Entscheidung darüber, wie eine bestimmte Information publiziert wird. ${ }^{19}$ Dass Medien als gewinnstrebige Unternehmen Informationen veröffentlichen, steht einem Schutz durch die Kommunikationsgrundrechte nicht entgegen. Al-

$18 \overline{\text { Tercier Pierre, Le nouveau droit de la personna- }}$ lité, Zürich 1984, N 671.

19 Vgl. BGE 120 Ib 142 E. 4; Kley Andreas/Tophinke Esther, Kommentar zu Art. 16 BV, in: Ehrenzeller Bernhard/Schindler Benjamin/Schweizer Rainer J./Vallender Klaus A. (Hrsg.), Die schweizerische Bundesverfassung, St. Galler Kommentar, 3. Aufl., Zürich/St. Gallen 2014, Art. 16 BV N 12. 
lerdings handelt es sich dabei um private Interessen der Medienunternehmen. Daneben können sich Medien auch auf ihren Informationsauftrag und damit auf öffentliche Interessen berufen. Diese lassen sich unter dem Begriff des Informationsinteresses zusammenfassen. ${ }^{20}$

\section{b) Boulevard-Prominenz}

36 Das Bundesgericht führt in seinen Erwägungen zum Vorliegen einer Persönlichkeitsverletzung aus, trotz der Stellung des Klägers als Boulevard-Prominenter sprenge die Kampagne den Rahmen dessen, was sich der Beschwerdeführer gefallen lassen müsse (E. 6.5). Es bezieht sich dabei auf die langjährige Praxis betreffend Personen des öffentlichen Lebens. ${ }^{21}$ Die Terminologie ist unterschiedlich. Häufig ist von absoluten oder relativen Personen der Zeitgeschichte die Rede. Das Bundesgericht geht davon aus, dass zudem ein Zwischenbereich besteht, dem es auch den Kläger zuordnet. ${ }^{22}$

Das Argument knüpft am öffentlichen Interesse an der Berichterstattung über eine Person an. Dieses kann dazu führen, dass die fraglichen Personen Abstriche bei ihrer Privatsphäre hinnehmen müssen. Obschon das Bundesgericht den Punkt in Bezug auf das Vorliegen einer Persönlichkeitsverletzung prüft, wäre er der Rechtfertigung einer Persönlichkeitsverletzung zuzuordnen. ${ }^{23}$ Auch in Bezug auf eine Persönlichkeitsverletzung durch eine Medienkampagne ist demnach zu prüfen, ob die betroffene Person aufgrund ihrer gesellschaftlichen Stellung eine solche Kampagne hinnehmen muss

Dazu Bacher (Fn. 10), N 680.

Vgl. BGE 127 III 481 E. 2.

Hirschmann I, E. $5.6 \mathrm{f}$.

BGE 127 III 481 E. 2c/aa. oder nicht. Je bedeutsamer eine Person, umso eher kann auch eine solche sehr intensive Berichterstattung gerechtfertigt sein. Unklar bleibt aufgrund des vorliegenden Urteils, ob der Bekanntheitsgrad einer Person alleine ein Rechtfertigungsgrund für eine Medienkampagne darstellen könnte.

\section{c) Überwiegendes öffentliches Interesse}

38 Die Besonderheit einer Persönlichkeitsverletzung durch eine Medienkampagne liegt darin, dass die Verletzung primär durch bestimmte Handlungen ausgelöst wird, die zu einer tatsächlichen und spürbaren Beeinträchtigung führen. Die Verletzung entsteht damit nicht durch bestimmte Inhalte, sondern durch die Art und Weise der Veröffentlichung. Dies ist bei der Abwägung zu berücksichtigen. Es muss mithin ein öffentliches Interesse an dieser Form der Publikation vorliegen, nicht einfach daran, die fraglichen Inhalte zu veröffentlichen. ${ }^{24}$ Ein solches Interesse kann durchaus gegeben sein, beispielsweise wenn es darum geht, gegen starken (z.B. politischen) Widerstand auf schwerwiegende Missstände aufmerksam zu machen.

39 Zentral ist darüber hinaus, auch bei der Abwägung zur Rechtfertigung einer Persönlichkeitsverletzung durch eine Medienkampagne die sich gegenüberstehenden Interessen neutral zu betrachten. Da die Kommunikationsgrundrechte auch die freie Wahl der Publikationsform schützen, darf es nicht per se ausgeschlossen sein, eine Medienkampagne zu rechtfertigen. Insgesamt muss die einschneidende Form der Berichterstattung

24 Vgl. dazu BGE 126 II 305 E. 4a. 
mit Blick auf den Inhalt der Information und das Ziel der Publikation angemessen sein, damit die Persönlichkeitsverletzung gerechtfertigt erscheint.

40 Das Bundesgericht nimmt diese Abwägung in E. 6.7.3 vor. Es relativiert dabei seine ständige Rechtsprechung, wonach eine Rechtfertigung stets nur so weit reichen könne, als ein Informationsbedürfnis bestehe, indem es ausführt, auch Sensationsberichte könnten im öffentlichen Interesse der Unterhaltung liegen.

Unklar bleibt jedoch, ob das Gericht eine Medienkampagne stets dem Sensationsjournalismus zuordnet. Dies lässt insbesondere der einleitende Satz vermuten, wonach eine Medienkampagne die «kollektive Klatschsucht» bediene. Eine solche Perspektive würde einer neutralen Gegenüberstellung der Interessen entgegenlaufen und wäre darüber hinaus gar nicht nötig. Denn die Frage ist nicht, ob eine Medienkampagne per se verwerflich ist, sondern ob sie im vorliegenden Kontext gerechtfertigt ist. Dazu grenzt das Bundesgericht die reine Unterhaltung von der Informationsvermittlung ab. Es orientiert sich damit an der Rechtsprechung des EGMR, der jeweils prüft, ob eine Information einen Beitrag zu einer Diskussion von allgemeinem Interesse leistet. 25

Das Bundesgericht kommt dabei zum Schluss, dass es vorliegend nicht um die Informationsvermittlung gegangen sei (Klarheit verschaffen über den Gang des Strafverfahrens), sondern um «Beteili- gung am Medienrummel» sowie das Aufrechterhalten «des medialen Karussells» und damit um ein reines Unterhaltungsbedürfnis. Dieses vermöge die Persönlichkeitsverletzung durch die Kampagne nicht aufzuwiegen.

\section{d) Überwiegendes privates Interesse}

43 Das Bundesgericht lässt es in seinem Entscheid offen, ob rein wirtschaftliche Interessen der Beklagten die Persönlichkeitsverletzung $\mathrm{zu}$ rechtfertigen vermöchten, weil die Beklagten auch kein solches behauptet hatten. Eine Rechtfertigung mittels wirtschaftlicher Interessen wäre indes auch kaum denkbar. Es würde bedeuten, dass der Kläger eine Persönlichkeitsverletzung durch eine Medienkampagne hinnehmen müsste, weil die Medien damit ihre Verkaufszahlen erhöhen können. Das wäre wenig sachgerecht. Der Nachweis eines solchen Interesses dürfte zudem schwerfallen, zumal sich die Medien in Bezug auf die Gewinnherausgabe regelmässig darauf berufen, es liesse sich kaum eruieren, ob ein bestimmter Artikel tatsächlich zu einer Gewinnsteigerung geführt habe. ${ }^{26}$

\section{Negatorische Klagen}

44 Gegen eine widerrechtliche Persönlichkeitsverletzung sind nach Art. 28a Abs. 1 ZGB die Unterlassungs-, Beseitigungsund Feststellungsklage zulässig. Zu prüfen ist, ob diese auch im Zusammenhang mit einer Persönlichkeitsverletzung durch eine Medienkampagne zur Anwendung kommen können.
25 Vgl. z.B. EGMR 40660/08 (v. Hannover/ Deutschland) vom 7.2.2012 (Caroline II), Ziff. 109; EGMR 59320/O0 (v. Hannover/Deutschland) vom 24.6.2004 (Caroline I), Ziff. 63 ff.
26 Vgl. z.B. Schwaibold (Fn. 3), N 32. 
Das Bundesgericht stellt in E. 6.8 eine widerrechtliche Persönlichkeitsverletzung durch eine Medienkampagne betreffend die Verhaftung des Klägers fest und heisst damit die Feststellungsklage gut. Die Feststellung einer Persönlichkeitsverletzung durch eine Medienkampagne scheint insgesamt unproblematisch.

Der Kläger erhebt darüber hinaus eine Beseitigungsklage. Erfolgt die Persönlichkeitsverletzung durch eine Medienkampagne, bezieht sich konsequenterweise auch die Beseitigungsklage auf die Kampagne und nicht auf einzelne Berichte. Dass die Berichte, welche Teil der Medienkampagne waren, nach wie vor im Internet abrufbar sind, bedeutet für das Bundesgericht aber nicht, dass die Medienkampagne noch andauert. Diese Überlegung ist zwar folgerichtig. Sie dürfte aber wohl bedeuten, dass eine Beseitigungsklage in Bezug auf eine persönlichkeitsverletzende Medienkampagne nur erfolgreich sein kann, wenn die Kampagne zum Zeitpunkt der Klageerhebung noch in vollem Gange ist. Denn die Beseitigungsklage hat in diesem Kontext das Ziel, eine andauernde Medienkampagne zu unterbrechen. Fraglich ist, ob diese Überlegung bei der Prosequierung vorsorglicher Massnahmen gegen eine Medienkampagne zu Schwierigkeiten führt.

Eine Unterlassungsklage gegenüber einer Persönlichkeitsverletzung durch eine Medienkampagne ist hingegen schwer vorstellbar. Wie sollte eine Person vorab wissen, dass ein Medium plant, über eine längere Zeit Inhalte zu einem beschränkten Thema über sie zu veröffentlichen?

\section{Ergebnis}

48 Die vorangehenden Ausführungen zeigen, dass eine Verallgemeinerung der Persönlichkeitsverletzung durch eine Medienkampagne tragfähig ist. Das Urteil gehört damit zu einer Reihe von Entscheiden, mit denen das Bundesgericht den zivilrechtlichen Persönlichkeitsschutz weiterentwickelt, um ihn den Gegebenheiten der neueren Medienlandschaft anzupassen. ${ }^{27}$ So bezieht sich das Gericht in E. 6.4.3 explizit auf die Digitalisierung und die Gefahren, welche deren Möglichkeiten für die betroffenen Personen mit sich bringen können.

49 Der umstrittenste Punkt des Entscheids dürfte sein, dass das Bundesgericht an die informationelle Selbstbestimmung anknüpft. Allerdings relativiert es dies, indem nicht alleine die Handlung, sondern die tatsächliche und spürbare Beeinträchtigung massgebend ist. Mit Blick auf eine Medienkampagne scheint es einleuchtend, mit dem Selbstbestimmungsrecht zu argumentieren. Es wird sich zeigen, ob künftig auch bei Publikationen, die keine Kampagnen im Sinne des Urteils darstellen, hauptsächlich auf die informationelle Selbstbestimmung abgestellt wird.

5o Selbst mit der vorliegenden Relativierung der informationellen Selbstbestimmung besteht dabei die Gefahr, dass die sehr differenzierten, inhaltsbezogenen Abwägungskriterien aus der bisherigen Praxis an Bedeutung verlieren. So wäre bei der Berichterstattung über die Privat- oder Geheimsphäre wohl stets von einer tat-

27 Vgl. u.a. BGE 133 III 153; Urteil des Bundesgerichts 5A_792/2011 vom 14. Januar 2013; Urteil des Bundesgerichts 5A_605/2007 vom 4. Dezember 2008. 
sächlichen und spürbaren Beeinträchtigung auszugehen. Dabei entfiele aber die oft hilfreiche Abgrenzung von Privatund Geheimsphäre. Das würde auch die Abwägung beeinflussen, die - wie auch das Urteil zeigt - primär an inhaltliche Kriterien anknüpft. Dies scheint im $\mathrm{Zu}-$ sammenhang mit der Medienberichterstattung sachgerecht. Auch sollte die Rechtsprechung zu Art. 28 ZGB dogmatische Fragen sowie Umsetzungsschwierigkeiten aus dem Bereich des Datenschutzes nicht übernehmen.

Insgesamt scheint es daher sinnvoll, nur in Bezug auf die Medienkampagne hauptsächlich auf die informationelle Selbstbestimmung abzustellen. Ebenfalls werden weitere Urteile deren Tatbestandsmerkmale noch konkretisieren müssen. So bleibt es nach dem vorliegenden Entscheid noch recht vage, wann eine Berichterstattung die Schwelle zur Medienkampagne überschreitet. Wichtig ist, dass es sich dabei um eine qualifizierte Form der Medienberichterstattung handeln sollte.

Eine «Katastrophe» stellt es aber meiner Ansicht nach nicht dar, wenn die Medien künftig etwas stärker überlegen müssen, ob bereits die Form der Publikation zu einer Persönlichkeitsverletzung führt. Das hätten sie nämlich bereits nach der bisherigen Rechtsprechung tun sollen. Denn obschon die freie Wahl der Form durch die Kommunikationsgrundrechte garantiert ist, schützen diese doch primär die Publikation von Inhalten und nicht bestimmte mediale Geschäftsmodelle.
53 Unbegründet scheint auch die Befürchtung von Schwaibold ${ }^{28}$, das Urteil bedeute das Ende des kritischen Journalismus. In Hirschmann II dürfte es wohl kaum um investigativen Journalismus gegangen sein. Vor allem aber lassen sich die vorgenannten Kriterien so auslegen, dass kritisch investigative Berichterstattung nicht unter den Begriff der Medienkampagne fällt, insbesondere weil sie thematisch anders angelegt ist. Selbst wenn diese Art von Berichterstattung als Persönlichkeitsverletzung durch eine Medienkampagne eingestuft würde, werden inhaltsbezogene Kriterien, insbesondere das Informationsinteresse, bei der Abwägung berücksichtigt. Liegt tatsächlich investigativer Journalismus vor, wird das Informationsinteresse regelmässig überwiegen und die betroffene Person muss die Kampagne hinnehmen.

54 Noch nicht ganz geklärt scheint jedoch das Verhältnis der Persönlichkeitsverletzung durch eine Medienkampagne zu persönlichkeitsverletzenden Inhalten.29 Zwar weist das Bundesgericht folgerichtig die Beseitigungsklage $a b$, mit denen der Kläger die gesamten Berichte, aus denen die Medienkampagne bestand, beseitigen lassen wollte. Zugleich heisst das Gericht aber Beseitigungs- und Feststellungsklagen zu einzelnen Berichten gut, welche wohl ebenfalls Bestandteil der Medienkampagne waren. Die Schaffung solcher «doppelrelevanter Tatsachen» erscheint jedoch nicht ideal, zumal auch zu klären wäre, ob dies auch zu einer Kumulation der reparatorischen Ansprüche führen kann. Das Bundesgericht scheint in E. 8.2.2.3 in diese Richtung zu gehen.

28 Schwaibold (Fn. 3), N 22.

29 Dazu auch Schwaibold (Fn. 3), N 9, der von einem doppelten Feststellungsanspruch spricht. 


\section{Schlusswort}

55 Beim Urteil Hirschmann II handelt es sich ohne Zweifel um ein Grundsatzurteil für den zivilrechtlichen Persönlichkeitsschutz. Durch die Bezugnahme auf den Datenschutz dürfte es auch für andere Bereiche bedeutsam werden, nicht zuletzt als Konkretisierung grundrechtlicher Ansprüche des Persönlichkeitsschutzes.

56 Wie dargestellt, erweist sich die Verallgemeinerung der Persönlichkeitsverletzung durch die Medien als tragfähig. Einzelne Punkte bleiben dabei allerdings noch unklar. Das Bundesgericht wird sicherlich Gelegenheit haben, sich in weiteren Urteilen dazu zu äussern, wobei nicht auszuschliessen ist, dass es sich dabei um einen Entscheid Hirschmann III handeln könnte. 